\title{
Medication review and patient counselling at discharge from the hospital by community pharmacists
}

\author{
J. G. Hugtenburg $\cdot$ S. D. Borgsteede • \\ J. J. Beckeringh
}

Received: 25 November 2008/ Accepted: 29 June 2009/Published online: 1 August 2009

(C) Springer Science+Business Media B.V. 2009

\begin{abstract}
Aims In 2001, the Association of Amsterdam Community Pharmacists adopted a programme to improve the pharmaceutical care of patients who were discharged from hospital with five or more drug prescriptions. A comprehensive protocol for pharmaceutical care at discharge (IBOM-1) was developed. The aim of the study was to evaluate the initial IBOM protocol and to study the effects of the protocol on drug therapy and patient satisfaction as well as on drug use compliance and mortality. Method A controlled intervention study involving 37 community pharmacies and 715 of their registered patients who were discharged from a hospital and using at least five prescribed drugs in the years 2001-2003. The intervention included an extensive medication review and drug counselling at the patient's home. Main outcome measure Pharmacy intervention activities, changes in medication, discontinuation of drugs prescribed at discharge, mortality, time spent on the intervention activities, and medication cost savings were all evaluated. Patient satisfaction was measured by means of a questionnaire. Results 379 and 336
\end{abstract}

J. G. Hugtenburg ( $($ )

Department of Clinical Pharmacology and Pharmacy,

VU University Medical Center, De Boelelaan 1117,

$1081 \mathrm{HV}$, Amsterdam, The Netherlands

e-mail: JG.Hugtenburg@vumc.nl

S. D. Borgsteede

Department of Hospital Pharmacy, Sint Lucas Andreas Hospital,

Amsterdam, The Netherlands

J. J. Beckeringh

Dutch Health Insurance Board, Diemen, The Netherlands patients were enrolled in the intervention and control groups, respectively. The mean number of drugs per patient not dispensed, concomitantly dispensed, or of which the quantity was changed was higher in the intervention group than in the control group $(0.70 \pm 1.74$ vs. $0.40 \pm 1.43$, $0.11 \pm 0.40$ vs. $0.038 \pm 0.26$, and $0.29 \pm 1.05$ vs. $0.097 \pm 0.52$, respectively). The mean number of drugs for which the dose or dosage form was changed was similar in both groups. Substitution of brand for generic or vice versa was greater in the intervention group. Changes resulting from a PAIS signal were similar in both groups. The mean number of drugs per patient for which contact was required with the physician or the Pharmacy Hospital Service Desk was higher in the intervention group $(0.35 \pm 0.51$ vs. $0.16 \pm 0.38$ ). About $40 \%$ of home visits resulted in the clearing of redundant drug supplies. The IBOM-1 intervention did not influence discontinuation of drugs prescribed at discharge, nor did it influence mortality. Medication costs were slightly reduced. More patients of intervention pharmacies than of control pharmacies indicated that they were (very) satisfied with the drug counselling by their community pharmacist upon delivery of their discharge medication $\left(87 \%\right.$ vs. $\left.50 \% ; \chi^{2}<0.001\right)$. Conclusions Structured pharmaceutical care according to the IBOM-1 protocol led to more changes in drug therapy. Home visits resulted in the clearing of redundant home drug supplies. In addition, patients were highly satisfied with the counselling at discharge from hospital by their community pharmacist. Patient counselling at discharge from hospital by pharmacists, therefore, appears to be a meaningful pharmaceutical care activity.

Keywords Discharge from hospital - Drug counselling . Medication review - Patient satisfaction - Persistence . Polypharmacy · The Netherlands 


\section{Impact of findings on practice}

- IBOM-1 resulted in more changes in drug therapy.

- Home visits resulted in the clearing of redundant home drug supplies.

- Patients were satisfied with pharmaceutical care according to IBOM-1.

\section{Introduction}

Drug-related problems (DRPs), such as contraindications, interactions, adverse drug reactions (ADRs) and inefficacy of treatment, result from the specific drug effects in patients, as well as from causes such as prescription errors and noncompliance with treatment $[1,2]$. In many cases, DRPs are the underlying cause of hospital admissions [3]. Data in the literature suggest that $3-7 \%$ of hospitalisations are the result of DRPs [4-6].

Beyer and De Blaey [7] have calculated that, in the Netherlands, DRPs are causing 90.000 preventable hospitalisations each year. A large Australian study, furthermore, reported a prevalence rate of 0.67 for serious ADRs and $0.97 \%$ for other drug-related hospital admissions. Around a third of these events were considered preventable [8].

A systematic review of intervention studies showed that 38 studies aimed at a reducing DRPs caused excess morbidity, hospital admissions, and/or mortality [9]. In 17 studies, interventions were initiated by the pharmacist, while in eight studies, other primary healthcare professionals initiated the intervention. Thirteen studies concerned complex interventions, including (a component of) medication review aimed at reducing the risk of falls in the elderly.

This analysis of the literature suggests that pharmacistled interventions were effective in reducing hospital admissions. However, when the analysis was limited to randomised, controlled trials, the benefit was lost. Pooling the results of studies in the other categories showed no significant effects. Royal et al. [9], therefore, concluded that there is only weak evidence for the effectiveness of pharmacist-initiated medication review in reducing hospital admissions.

In an intervention study aimed at identifying DRPs during and after hospitalisation, Schnipper et al. [10] observed that medication review by a pharmacist, patient counselling, and follow-up by telephone were associated with a lower rate of DRPs 30 days after discharge. In a recent, large intervention study, $\mathrm{Wu}$ and colleagues [11] concluded that telephone counselling by pharmacists of polypharmacy patients who did not adhere to their medication schemes reduced mortality.
A hospital stay often leads to changes in the drug therapy of patients with chronic diseases [12]. Most patients are older people who are often using drugs that have been prescribed by general practitioners (GPs) as well as by specialist physicians. Hospital specialists may add new drugs and cancel the use of existing drugs, while patients may still have their cancelled drugs at home [12]. Once at home, therefore, it is not always clear to the patient which drugs should be used and how they should be used.

In the Netherlands, discharge prescriptions are supplied by a community pharmacy. Usually, the discharge prescription is sent directly from the hospital to the pharmacy where the patient is registered. This implies that the pharmacy implements all changes and informs the patient about the drugs they should use after discharge. An important tool in this process is the pharmacy administration and information system (PAIS), in which both patient and medication data are electronically stored. The automated processing of prescriptions includes a relatively extensive (but still limited) check on contraindications and interactions [13]. Warning signals of the PAIS are, therefore, an important contributor to reducing the number of possible and often preventable DRPs $[3,8,14]$. However, using a combination of structured and patient interactive interventions may be more effectively in preventing DRPs. Examples include: checking if discharge medication matches concomitant medication which has not been influenced by the hospital stay, interactive counselling of patients and/or carers regarding the effects, side effects and usage of drugs prescribed at discharge, and checking whether any stock of cancelled and/or redundant drugs is handed in by the patient/carer.

In 2001, the Association of Amsterdam Community Pharmacists adopted a programme to improve the pharmaceutical care of patients who were discharged from hospital with five or more drug prescriptions. As an initial step, a comprehensive protocol was developed, as shown in Table 2. The aim of the study was to evaluate the initial IBOM protocol and to study the effects of the protocol on drug therapy and patient satisfaction as well as on drug use compliance and mortality.

\section{Method}

Study design

This study is based on a controlled intervention study involving 37 community pharmacies and 715 patients discharged from hospital over the years 2001-2003. Control pharmacies provided usual care, shown in Table 1, at the level of the then current version of the Dutch National Pharmacy Standard. Intervention pharmacies provided 
Table 1 Delivery of medication at discharge in 2001-2003 (A) and usual care, according to the Dutch Pharmacy Standard ${ }^{\mathrm{a}}$ (B) [15]

A1. In the Amsterdam area, in each of its six major hospitals, the Pharmacy Service Desk (PSD) routinely sends a discharge prescription by fax to the community pharmacy where the patient is registered.

A2. Prescriptions were in the form of a list of drugs that should be dispensed to the patient, a list of drugs prescribed in the hospital. The use of which should be continued or in the form of an overview of all medication that the patient should use. In the latter case, it was indicated which drugs should be dispensed. The type of prescription depended on the hospital and/or the hospital department to which the patient was admitted.

B1. Preceding the release check, prescriptions are routinely checked for drug interactions and contra-indications by the PAIS.

B2. Discharge medication is delivered at the patient's home or is picked up by the patient or carer in the pharmacy.

B3. Drugs are routinely delivered with a drug information leaflet, but patients are also often handed supplementary personalised PAIS-generated information letters with newly prescribed drugs.

B4. When the discharge medication is collected from the pharmacy, the patient or carer is also provided with additional oral information about newly prescribed drugs. This includes an explanation of the drugs' actions, their use and of possible side effects.

${ }^{a}$ First edition of 1996. The current (2nd) edition was introduced in 2006 (KNMP, The Hague)

Table 2 The IBOM-1 intervention protocol

1. Review of the medication record of the patient by the pharmacist after receipt of the discharge prescription and initial processing at level 1, as described by the Task Force on Medicines Partnership and The National Collaborative Medicines Management Services Programme [16]. This includes a comparison of the drugs on the discharge prescription with all medication used before the hospital admission. The review also takes account of possible home supplies.

2. Home visit by the pharmacist within 1 week after delivery of the discharge medication.

3. All patients are given a printed or handwritten overview of their medication and a daily medication intake scheme (weekly basis).

4. A copy of the latter document is sent to their GPs.

5. All drugs must be synchronised for a similar period of time.

6. Check of home supplies.

extended care according to the IBOM-1 protocol, as seen in Table 2.

\section{Pharmacies}

All pharmacies in the Amsterdam area were invited to participate. Pharmacists could indicate whether they preferred to participate as an intervention pharmacy or a control pharmacy.

\section{Patients}

Each pharmacy was asked to include 20 patients who were successively discharged from hospital with at least five prescribed drugs. Exclusion criteria were discharged to a nursing home, inability to understand Dutch, and mental illness. The number of patients was not based on power calculations, but on feasibility of the intervention activities within daily pharmacy practice.

\section{Pharmacy data processing}

Discharge prescriptions were routinely entered into the PAIS and the electronic medication record of each patient. Data from the medication record were either extracted in the form of a medication profile spanning a pre-specified period, or used to produce daily medication intake schemes or personalised patient information letters.

\section{Privacy}

The Institutional Review Board did not consider the study to such an extent invasive of the participants' integrity that review by the Board was necessary. All data were processed in accordance with Dutch privacy regulations. Patients had to give their written consent. Each patient was given a randomly assigned, unique number, which was made anonymous in the questionnaires.

\section{Measurements}

\section{Pharmacy interventions in the discharge prescription}

All drugs prescribed to the patient at discharge were compared with the drugs the patients used prior to hospitalisation. The following differences were recorded on study forms: issuing of additional drugs not listed on the discharge prescription; cancellation of a drug; change in quantity; change of dose; change of dosage form, substitution (brand for generic or vice versa); change of medication as the result 
of a PAIS warning signal; and contact with a physician (specialist and/or GP) or Hospital Pharmacy Service Desk.

\section{Performance of other intervention activities}

Pharmacists' other intervention activities were also recorded on study forms developed specifically for use in the intervention pharmacies. These included: making an overview of the medication record (medication passport); producing a daily medication intake scheme; sending a copy of these documents to the patient's GP; synchronising discharge and concomitant medication on time; interviewing the patient (at home/in the pharmacy/by telephone); and checking home supplies of drugs.

\section{Patient satisfaction}

Patients' satisfaction with the drug counselling by their pharmacist was studied by means of sending a questionnaire to each patient 6-9 months after their discharge. Patients were asked to what extent they were satisfied with the delivery of drugs and drug counselling by their pharmacist. Patients could reply to the questions on a five-point Likert scale.

\section{Discontinuation of drugs newly prescribed at discharge}

Nine months after hospital discharge, the medication record was reviewed with the aim to assess whether drugs for treatment of a chronic disease first prescribed at discharge were still being used.

\section{Mortality}

The mortality rate 9 months after discharge from the hospital was determined on the basis of patient data extracted from the PAIS.

\section{Time spent on patient counselling and costs saved}

The time spent on patient counselling, the cost of supplemental drugs and/or costs saved by reducing the amount of drugs dispensed at discharge were also recorded.

\section{Data collection and analysis}

After the inclusion and counselling of 20 successive patients, the study forms and copies of the daily medication intake schemes and discharge prescriptions were collected. Overviews of drugs dispensed over a period of 9 months following discharge were provided by each pharmacy. Data were collected and analysed in SPSS 10.0. Students' $t$-test and $\chi^{2}$-test were used. $P<0.05$ was considered significant.

\section{Results}

Basic characteristics

The intervention and control groups included 336 and 379 patients respectively. Basic characteristics of the patients are shown in Table 3. The male/female distribution was similar in both groups. Only the patients in the intervention group were slightly younger and the mean number of drugs prescribed was higher for this group. In both the intervention group, as the control group, no data were missing with respect to the basic characteristics and the intervention measurements. The only exception is patient satisfaction: questionnaires were received by 112 patients in the intervention group and 146 in the control group. Primary reasons were: patient death $(22 \%)$ and loss to follow-up.

Pharmacy interventions in the discharge prescription

Distribution of intervention measurement outcomes was skewed strongly to the left, with only few patients (less than $5 \%$ ) that had received more than one intervention of the same type. Data were dichotomised in patients with and without one or more pharmacy intervention. For the following interventions, the proportion of patients in whom one or more interventions was higher in patients in the intervention group than in the control group: individual drugs dispensed, drugs not dispensed, quantity changed of drugs that were actually dispensed change of dose, and contact with physician or Hospital Pharmacy Service Desk (Table 4).

Delivery of drugs and counselling

The delivery of drugs in relation to the intervention is shown in Table 5. Intervention pharmacists counselled the majority of patients at home $(60 \%)$. Yet, a substantial number of patients were counselled in the pharmacy $(19 \%)$ or by telephone (14\%).

Table 3 Patient characteristics

\begin{tabular}{|c|c|c|c|}
\hline & $\begin{array}{l}\text { Intervention } \\
\text { group }\end{array}$ & $\begin{array}{l}\text { Control } \\
\text { group }\end{array}$ & $P$ \\
\hline Number of patients & 336 & 379 & \\
\hline Male/female & $164 / 172$ & $177 / 202$ & 0.317 \\
\hline Age, mean \pm SE & $69.7 \pm 15.0$ & $72.7 \pm 11.2$ & 0.004 \\
\hline $\begin{array}{l}\text { Number of drugs prescribed per } \\
\text { patient, mean } \pm \text { SE }\end{array}$ & $7.8 \pm 2.6$ & $7.1 \pm 2.3$ & $<0.001$ \\
\hline $\begin{array}{l}\text { Patients from cardiologic, } \\
\text { internal, and pulmonary } \\
\text { departments }(\%)\end{array}$ & 81.6 & 83.7 & 0.545 \\
\hline
\end{tabular}


Table 4 Pharmacy interventions on discharge prescriptions

\begin{tabular}{|c|c|c|c|c|c|}
\hline & \multicolumn{2}{|c|}{ Intervention group } & \multicolumn{2}{|c|}{ Control group } & \multirow[t]{2}{*}{$P$-value $\chi^{2}$} \\
\hline & $\begin{array}{l}\text { Number of } \\
\text { patients }(\%)\end{array}$ & $\begin{array}{l}\text { Mean number per } \\
\text { patient } N=336\end{array}$ & $\begin{array}{l}\text { Number of } \\
\text { patients }(\%)\end{array}$ & $\begin{array}{l}\text { Mean number per } \\
\text { patient } N=379\end{array}$ & \\
\hline Individual drugs not dispensed & $80(23.8)$ & $0.70 \pm 1.74$ & $53(14.2)$ & $0.40 \pm 1.43$ & 0.001 \\
\hline Additional drugs dispensed & $30(8.9)$ & $0.11 \pm 0.40$ & $11(2.9)$ & $0.038 \pm 0.26$ & 0.001 \\
\hline Quantity changed of drugs that were actually dispensed & $42(14.3)$ & $0.29 \pm 1.05$ & $22(5.9)$ & $0.097 \pm 0.52$ & $<0.001$ \\
\hline Change of dose & $44(13.1)$ & $0.14 \pm 0.38$ & $29(7.8)$ & $0.094 \pm 0.35$ & 0.020 \\
\hline Change of dosage form & $16(4.8)$ & $0.06 \pm 0.28$ & $18(4.8)$ & $0.051 \pm 0.23$ & 0.968 \\
\hline Substitution brand/generic or vice versa & $95(28.3)$ & $0.60 \pm 1.22$ & $97(26.0)$ & $1.21 \pm 2.52$ & 0.497 \\
\hline Change of medication as the result of PAIS signal & $28(8.3)$ & $0.60 \pm 1.22$ & $28(7.5)$ & $1.21 \pm 2.52$ & 0.684 \\
\hline Contact with physician or Hospital Pharmacy Service Desk & $112(33.3)$ & $0.35 \pm 0.51$ & $60(16.1)$ & $0.16 \pm 0.38$ & $<0.001$ \\
\hline
\end{tabular}

Table 5 Delivery of drugs and counselling

\begin{tabular}{|c|c|c|c|c|}
\hline & \multicolumn{2}{|c|}{ Intervention group $N=336$} & \multicolumn{2}{|c|}{ Control group $N=379$} \\
\hline & Number & $\%$ & Number & $\%$ \\
\hline Delivered at home, no counselling & & & 277 & 73.1 \\
\hline Delivered at home before counselling visit & 38 & 11.3 & & \\
\hline Delivered at home, counselling by telephone & 46 & 13.7 & & \\
\hline Delivered at home together with counselling & 163 & 48.5 & 60 & 15.8 \\
\hline Delivery and counselling in the pharmacy & 62 & 18.5 & 12 & 3.2 \\
\hline Delivery in pharmacy, no counselling & 27 & 8.0 & 30 & 7.9 \\
\hline
\end{tabular}

A small number of patients $(8 \%)$ were not counselled. In the intervention group, 133 patients (39.6\%) mentioned having a problem with their medication or raised questions about it. In the control group, 72 patients (19\%) were counselled as part of the usual care process. Some pharmacists in the control group also visited their patients at home to give some form of counselling (16\%) or did the counselling when patients collected their medication in the pharmacy (3\%), shown in Table 5. In this group, 38 patients $(10 \%)$ mentioned having a problem with their medication or raised questions about it.

\section{Evaluation of intervention activities}

The pharmacists' activities concerning the execution of the intervention are shown in Table 6. Not all intervention activities were fully implemented by the pharmacists. Most patients $(83 \%)$ were given a printed daily medication intake scheme. A much smaller number (38.7\%) was also given a medication overview in the form of a 'medication passport'. In most cases (78\%) GPs were also informed. Supplies of redundant drugs were taken in from well over $40 \%$ of patients counselled at home. In the case of 54 patients $(16 \%)$ the initial IBOM-1 protocol intervention was followed by contacting the patient at a later stage. Follow-up to the initial counselling was given to 66
Table 6 Overview of pharmacy intervention activities

\begin{tabular}{lll}
\hline & \multicolumn{2}{l}{ Intervention group $N=336$} \\
\cline { 2 - 3 } & Number & $\%$ \\
\hline Medication overview produced & 130 & 38.7 \\
$\begin{array}{l}\text { Daily medication intake scheme } \\
\text { handed out }\end{array}$ & 278 & 82.7 \\
$\quad$ Medication synchronised & 37 & 11.0 \\
Taking in home supplies of drugs & 86 & $42.8^{\mathrm{a}}$ \\
Any intervention activity at a later & 54 & 16.1 \\
$\quad$ stage & & \\
$\begin{array}{l}\text { Follow-up counselling } \\
\text { Copy of daily medication intake }\end{array}$ & 261 & $21.4^{\mathrm{b}}$ \\
$\quad$ scheme was sent to GP & & 77.7 \\
\hline
\end{tabular}

${ }^{a}$ Percentage of patients counselled at home

b Percentage of all patients counselled

patients (21\%). In $16(4.2 \%)$ control patients any intervention activity was performed.

\section{Patient satisfaction}

The percentage of patients of intervention and control pharmacies who were (very) satisfied about the drug counselling in the hospital was similar (46\%). More patients of intervention pharmacies, than of control 
pharmacies, indicated that they were very satisfied about the drug counselling upon delivery of their discharge medication by their community pharmacist. In the intervention patients $(n=112), 87 \%$ were very satisfied, the median score was 'very satisfied', range from neutral to very satisfied. In the control patients $(n=146), 50 \%$ were very satisfied, the median score was 'satisfied', range from dissatisfied to very satisfied. The difference was statistically significant $\left(\chi^{2}\right.$-test, $\left.P<0.001\right)$.

Discontinuation of drugs newly prescribed at discharge

Over a 9-month period after discharge from hospital, a few more patients of pharmacies in the intervention group than the control group ceased using the drugs that were first prescribed in hospital for the chronic disease for which they had been treated (64\% vs. 58\%). The difference, however, was not significant.

\section{Mortality}

At the end of the 9-month study period, there was no difference between the mortality of patients of intervention pharmacies and patients of control pharmacies $(22 \%$ in each group).

Time spent on patient counselling and costs saved

The time spent on patient counselling amounted to $26.3 \pm 15.7$ min per patient. Costs saved by reducing the amount of drugs delivered amounted to $19.5 \pm 47.9$ Euros per patient.

\section{Discussion}

With respect to the delivery of discharge medication, the results of the present study show that intervention by community pharmacists according to the IBOM-1 protocol leads to a higher rate of adjustments made to the quantity of drugs dispensed. It also leads to a higher frequency of contact between community pharmacists and hospitals. As we expected, there was no difference in the number of PAIS-generated warning signals that led to a change of pharmacotherapy. The number of adjustments made to prescribed doses and dosage forms were also similar. Although hospital prescriptions, at discharge, appeared to be technically correct, they did not take sufficiently into account the actual need of patients. Review of the medication showed that, in the majority of cases, patients already had sufficient stock of drugs that were not dispensed, rather than there being a pharmacotherapeutic reason for not dispensing these drugs. Thus, it appears that application of the IBOM-1 protocol improved the quality of the drug-dispensing procedure, which resulted in a reduced risk of accumulated supplies of (possibly redundant) drugs at the patient's home, and, to a lesser extent, in rationalisation of pharmacotherapy.

\section{Evaluation of the IBOM-1 protocol}

In the majority of cases, the medication prescribed at discharge was delivered to homes by the control pharmacies. This implies that most patients were only informed of the new drugs and their usage by means of the labels on the drug packages, the patient information leaflet and, occasionally, by a personalised information letter from the pharmacy. In only $20 \%$ of cases, an additional verbal explanation or counselling was given. Almost $40 \%$ of the patients of intervention pharmacies mentioned that they had some kind of problem in using their medication, or raised a specific question about their medication when visited at home. This suggests that the standard procedure for dispensing discharge medication is satisfactory. Yet, information given to patients is not.

The most important part of the IBOM-1 intervention is the counselling of patients at home, on the basis of an indepth review of their medication and a printed copy of the daily medication intake scheme for the period of 1 week for each patient. Indeed, $83 \%$ of patients of intervention pharmacies were given a daily medication intake scheme. As intended, $92 \%$ of patients were counselled, but only $60 \%$ were paid a home visit. This means that, in a considerable number of cases, there was no opportunity to check home supplies of prescription as well as non-prescription drugs.

According to the study data, in only $39 \%$ of cases was a medication overview or 'medication passport' produced in the intervention pharmacies. However, with the more important daily medication intake scheme available, the issuing of this document, which, as its name suggests, was initially conceived as a useful document for patients going on holiday abroad, might be considered redundant. For this reason, it was skipped by many pharmacies.

In view of the number of patients counselled and the number of daily medication intake schemes handed out to patients and sent to GPs, it can be concluded that, in the majority of cases, the medication has been reviewed and dispensed as intended by the IBOM-1 protocol. Around a fifth of the patients were not given a daily medication intake scheme. It is well possible that, in these cases, there had been no changes in the medication after discharge and, therefore, it was considered unnecessary to hand out a daily medication intake scheme. In relatively few cases, medication was synchronised. This low percentage may result from the earlier decision whether or not to dispense a drug, 
and the possibility that synchronisation was performed automatically on the basis of stock positions according to the PAIS.

When patients were visited at home, in about 40 percent of the cases, cancelled and/or redundant drugs were taken in. On many study forms, this item was not ticked off, which suggests that some pharmacists did not fully implement the intervention programme. In addition, it might not have been applicable to all patients. However, it should be acknowledged that checking patients' home supplies of drugs is very important, not only because unnecessary and redundant drugs may lead to confusion about which drugs should be used, which can result in various DRPs, but also because the use of any OTC drugs can thus be determined.

\section{Effectiveness of the IBOM-1 intervention}

The effectiveness of the intervention, with respect to DRPs and hospital admissions, has not been evaluated. However, with respect to continuation of drugs newly prescribed in the hospital, and mortality, the effect appears to be limited. A similar amount of patients discontinued the use of drugs for their chronic illness in the intervention and the control groups. This is consistent with Herings et al. [17], who also found low rates of persistence with chronic medication on the basis of data from a prescription database. Also, there was no effect on mortality.

Other studies [e.g. 18-23] have shown that home-based interventions provide a valuable tool for detecting problems that are likely to be a cause of poor health outcomes. The effects of these interventions on the occurrence of hospital admissions varied, and the interventions did not influence the number of readmissions [18, 20-23]. One of the factors that may account for the lack of effectiveness in our study might be the unstructured character of the medication review by the pharmacists, who were not trained for this specific task. In addition, a single home visit, as investigated in our study, might be insufficient. It has been shown that interventions using multiple home visits resulted in positive outcomes on multiple readmissions and the number of hospital days per patient [18]. However, intervention may also result in an increase of hospital admissions [22]. Home-based interventions might be particularly relevant for educational purposes, as patients are often more comfortable and prepared to learn at home [18, 19]. However, the differences in study objectives, settings, interventions, and outcomes make a detailed comparison difficult. With respect to a possible effect on mortality, it can be expected that a much larger number of participants is required to achieve sufficient power.
Study limitations

The participating pharmacies were not randomised. Although the basic characteristics of patients in both groups did not differ very much, the pharmacists that decided to participate as intervention pharmacists may have differed in some way from the pharmacists of control pharmacies. The basic pharmaceutical care given by an intervention pharmacist might already have been organised in a more structured fashion. Furthermore, it has not been checked by an independent observer whether changes made by pharmacists to the patients' drug therapy led to an improvement in the appropriateness of the drugs dispensed. In addition, the reasons for changes in drug regimen are not known. Also, information on patients' re-hospitalisations and DRPs was not recorded and cost-effectiveness was not studied in detail. These limitations of the study design are being addressed in the current IBOM-2 study.

\section{Recommendations}

DRPs, and specifically ADRs, form a major cause of the hospitalisation of patients. Polypharmacy, age of patients, and drug compliance appear determinants for hospitalisation resulting from ADRs. In this respect, interventions should be aimed at improving pharmacotherapy by means of effective strategies, including intensive medication review and stimulation of drug compliance. For this purpose, we have developed an intervention study, including medication review and patient counselling at discharge from hospital in combination, with a follow-up period of 1 year of patient counselling aimed at improving drug safety (IBOM-2). In this study, the effects on DRPs, patients' re-hospitalisation and their adherence to the drug regimen will be studied.

\section{Conclusion}

Structured pharmaceutical care according to the IBOM-1 protocol led to more changes in drug therapy. Home visits resulted in the clearing of redundant drug supplies. In addition, patients were highly satisfied with the counselling at discharge from hospital by their community pharmacist. Patient counselling at discharge from hospital by pharmacists, therefore, appears to be a meaningful pharmaceutical care activity. However, intervention by pharmacists should also be aimed at reducing DRPs and drug-related hospital admissions, by means of effective strategies, including structured medication review and improvement of drug adherence. These strategies will need to be supported by specific training of the pharmacists and by longitudinal contact between patients and their pharmacy after 
discharge from hospital. For a better understanding, outcomes such as the impact of intervention on drug adherence and changes in patients' attitudes toward their medication should be included in future studies.

Acknowledgements All pharmacists and patients who participated in the study are gratefully acknowledged for their assistance and cooperation.

Funding Funding of the study by AGIS health insurance company, Amsterdam, and WINap Dutch Scientific Institute of Pharmacists is gratefully acknowledged.

Conflict of interest statement None

\section{References}

1. Tam VC, Knowles SR, Cornish PL, Fine N, Marchesano R, Etchells EE. Frequency, type and clinical importance of medication history errors at admission to hospital: a systematic review. CMAJ. 2005;173:510-5.

2. DiMatteo MR. Variations in patients' adherence to medical recommendations: a quantitative review of 50 years of research. Med Care. 2004;42:200-9.

3. Winterstein AG, Sauer BC, Hepler CD, Poole C. Preventable drug-related hospital admissions. Ann Pharmacother. 2002; 36:1238-48.

4. Audit Commission. A prescription for improvement. Towards more rational prescribing in general practice. London: HMSO; 1994.

5. Runciman WB, Roughead EE, Semple SJ, Adams RJ. Adverse drug events and medication errors in Australia. Int J Qual Health Care. 2003;15(1):i49-59.

6. Lundkvist J, Johnsson B. Pharmacoeconomics of adverse drug reactions. Fundam Clin Pharmacol. 2004;18:275-80.

7. Beijer HJ, de Blaey CJ. Hospitalisations caused by adverse drug reactions (ADR): a meta-analysis of observational studies. Pharm World Sci. 2002;24:46-54.

8. Miller GC, Britt HC, Valenti L. Adverse drug events in general practice patients in Australia. MJA. 2006;184:321-4.

9. Royal S, Smeaton L, Avery A, Hurwitz B, Sheikh A. Interventions in primary care to reduce medication related adverse events and hospital admissions: systematic review and meta-analysis. Qual Saf Health Care. 2006;15:23-31.
10. Schnipper JL, Kirwin JL, Cotugno MC, Wahlstrom SA, Brown BA, Tarvin E, et al. Role of pharmacist counseling in preventing adverse drug events after hospitalization. Arch Intern Med. 2006;166:565-71.

11. Wu JY, Leung WY, Chang S, Lee B, Zee B, Tong PC, et al. Effectiveness of telephone counselling by a pharmacist in reducing mortality in patients receiving polypharmacy: randomised controlled trial. Br Med J. 2006; 333:522.

12. Kripalani S, Jackson AT, Schnipper JL, Coleman EA. Promoting effective transitions of care at hospital discharge: a review of key issues for hospitalists. J Hosp Med. 2007;2:314-23.

13. Buurma H, De Smet PA, Egberts AC. Clinical risk management in Dutch community pharmacies: the case of drug-drug interactions. Drug Saf. 2006;29:723-32.

14. Viktil KK, Blix HS. The impact of clinical pharmacists on drugrelated problems and clinical outcomes. Basic Clin Pharmacol Toxicol. 2008;102:275-80.

15. Dutch Pharmacy Standard, Second Edition 2006, Online. www.knmp.nl/download-bestanden/vakinhoud-1/kwaliteitszorg/ nan-en-richtlijnen/nan_2006.pdf.

16. Shaw J, Seal R, Pilling M. Room for review. A guide to medication review: the agenda for patients, practitioners and managers. London: Medicines Partnership; 2002.

17. Herings RMC, Leufkens HGM, Heerdink ER. Chronic pharmacotherapy continued. Utrecht: PHARMO Institute for PharmacoEpidemiological Research; 2002 (in Dutch).

18. Naylor MD, Brooten D, Campbell R, Jacobsen BS, Mezey MD, Pauly MV, et al. Comprehensive discharge planning and home follow-up of hospitalized elders: a randomized clinical trial. JAMA. 1999;281(7):613-20.

19. Ryder M, Travers B, Ledwidge M, McDonald K. Multidisciplinary care of heart failure: what have we learned and where can we improve? Eur J Cardiovasc Nurs. 2003;2(4):247-9.

20. Stewart S, Marley JE, Horowitz JD. Effects of a multidisciplinary, home-based intervention on unplanned readmissions and survival among patients with chronic congestive heart failure: a randomised controlled study. Lancet. 1999;354(9184):1077-83.

21. Nazareth I, Burton A, Shulman S, Smith P, Haines A, Timberal H. A pharmacy discharge plan for hospitalized elderly patientsa randomized controlled trial. Age Ageing. 2001;1:33-40.

22. Holland R, Lenaghan E, Harvey I, Smith R, Shepstone L, Lipp A, et al. Does home-based medication review keep older people out of hospital? The HOMER randomised controlled trial. Br Med J. 2005;330(7486):293.

23. Lenaghan E, Holland R, Brooks A. Home-based medication review in a high risk elderly population in primary care-the POLYMED randomised controlled trial. Age Ageing. 2007; 36:292-7. 